\title{
Associations between Neighborhood Walkability, Physical Activity, and Chronic Disease in Nova Scotian Adults: An Atlantic PATH Cohort Study
}

\author{
Melanie R. Keats ${ }^{1, *(\mathbb{D}}$, Yunsong Cui ${ }^{2}$, Vanessa DeClercq $\left.{ }^{2}{ }^{(}\right)$, Scott A. Grandy ${ }^{1}$, \\ Ellen Sweeney ${ }^{2}$ (i) and Trevor J. B. Dummer ${ }^{3}$ \\ 1 Faculty of Health, School of Health and Human Performance, Dalhousie University, \\ Halifax, NS B3H 4R2, Canada; grandy@dal.ca \\ 2 Atlantic Partnership for Tomorrow's Health, Faculty of Health, Dalhousie University, \\ Halifax, NS B3H 4R2, Canada; yunsong.cui@dal.ca (Y.C.); vanessa.de.clercq@dal.ca (V.D.); \\ ellen.sweeney@dal.ca (E.S.) \\ 3 School of Population and Public Health, University of British Columbia, Vancouver, BC V6T 1Z3, Canada; \\ trevor.dummer@ubc.ca \\ * Correspondence: melanie.keats@dal.ca; Tel.: +1-(902)-494-7173
}

Received: 29 October 2020; Accepted: 16 November 2020; Published: 20 November 2020

check for updates

\begin{abstract}
Background: While neighborhood walkability has been shown to positively influence health behaviors, less is known about its impact on chronic disease. Our aim was to examine the association between walkability and self-reported physical activity in relation to chronic health conditions in an Atlantic Canadian population. Methods: Using data from the Atlantic Partnership for Tomorrow's Health, a prospective cohort study, we employed both a cross-sectional and a prospective analytical approach to investigate associations of walkability and physical activity with five prevalent chronic diseases and multimorbidity. Results: The cross-sectional data show that participants with the lowest neighborhood walkability were more likely to have reported a pre-existing history of cancer and depression and least likely to report chronic respiratory conditions. Participants with low physical activity were more likely to have a pre-existing history of diabetes, chronic respiratory disease, and multimorbidity. Follow-up analyses showed no significant associations between walkability and chronic disease incidence. Low levels of physical activity were significantly associated with diabetes, cancer and multimorbidity. Conclusions: Our data provides evidence for the health protective benefits of higher levels of physical activity, and a reduction in prevalence of some chronic diseases in more walkable communities.
\end{abstract}

Keywords: neighborhood walkability; physical activity; chronic disease; cohort study

\section{Introduction}

As many as one-third of Canadian adults (20+ years) live with at least one of five common chronic diseases (i.e., cancer, diabetes, cardiovascular diseases, chronic respiratory diseases, and mood and/or anxiety disorders). Of these, $9 \%$ report having two or more of these chronic diseases [1]. Compared to other Canadians, Nova Scotians rank amongst the unhealthiest, with as many as $43 \%$ of the population reporting at least one and $11 \%$ reporting two or more of the five common chronic conditions [1]. With the highest proportion of Canadian seniors (65+ years; $17.2 \%$ ), coupled with a high prevalence of modifiable behavioral risk factors (e.g., physical inactivity, tobacco use, alcohol consumption, unhealthy diets), there is a critical need to identify strategies to reduce the health and economic burden of chronic disease amongst Nova Scotians [2-4]. 
Given that much of the chronic disease burden has been shown to be related to a relatively small number of modifiable behavioral risk factors, improving health behaviors is often a primary target for the prevention of chronic disease and improving health outcomes [5]. While much work has been focused on individual behavioral change, it is clear that the development of chronic disease is associated with many factors, and the management and prevention of chronic disease must include a multi-level/multi-sectorial approach [6]. Specifically, behavioral change does not occur in isolation, and health behaviors are influenced by the larger socioecological context in which an individual resides. For example, there is a considerable amount of evidence showing that neighborhood design and environmental features positively influence population health by supporting engagement in healthy behaviors (i.e., increased physical activity) $[7,8]$.

Composite measures of neighborhood walkability (i.e., design features that can promote walking and access to walkable destinations) have been consistently associated with a decreased risk of cardiometabolic diseases (e.g., obesity, hypertension, diabetes, and cardiovascular disease (CVD)) $[9,10]$. These protective effects are presumed to be partially attributed to increases in physical activity (i.e., higher walkability positively associated with physical activity in adults) [11]. To date, however, no Canadian study has examined the association between walkability and cancer, depression, or multimorbidity [8]. Moreover, no study has examined the association between standardized measures of walkability and health outcomes in any of the Atlantic Canadian provinces. Accordingly, the aims of this study were to (1) retrospectively examine the association between neighborhood walkability and self-reported physical activity in relation to five prevalent chronic health conditions (e.g., cancer, diabetes, cardiovascular disease, chronic respiratory disease, and depression) and multimorbidity in a sample of Nova Scotian adults, and (2) to prospectively examine incident disease risk in Nova Scotians residing in low-to-high walkability neighborhoods, while controlling for potential confounders.

\section{Methods}

\subsection{Design}

This study used data from the Atlantic Partnership for Tomorrow's Health (PATH). Atlantic PATH is part of the larger Canadian Partnership for Tomorrow's Health (CanPath; formerly the Canadian Partnership for Tomorrow Project), a national prospective cohort study examining the influence of genetic, environmental, and lifestyle factors on the development of chronic disease. A detailed description of the study has been previously described [12,13]. Briefly, from 2009 to 2015, 31,173 residents of the four Atlantic Canadian provinces (Nova Scotia, New Brunswick, Prince Edward Island, and Newfoundland) were invited to complete a standardized set of questionnaires designed to assess sociodemographic characteristics, health status, disease history, and lifestyle behaviors. Between 2016 and 2019, participants were invited to complete the first follow-up questionnaire aimed at updating previously collected data on sociodemographic, health, disease and lifestyle behaviors. The cross-sectional and prospective analyses in the current study are based on participants who resided in Nova Scotia at the time of data collection. The Atlantic PATH protocol was approved by the provincial and regional ethics boards, and all participants provided written informed consent prior to participation.

\subsection{Study Area and Population}

Nova Scotia is one of four Atlantic provinces located on Canada's east coast. It is the most populated province of the Atlantic region, with a population of 977,274 [14]. Halifax is the province's capital city and accounts for as much as $45 \%$ of the province's populace [15]. For the purposes of aim 1, the Atlantic PATH participants included in the baseline analyses $(N=15,215)$ were between the ages of 35 and 69 years old, living in Nova Scotia at the time of baseline assessment (2009-2015) with non-missing walkability and physical activity data. For aim 2, the Atlantic PATH participants included in the prospective analyses $(\mathrm{N}=6912)$ were living in Nova Scotia at both baseline and follow-up 
assessments (2016-2019), were residing in the same postal code area for at least two years, had not self-reported any of the five chronic diseases examined in aim 1, and had non-missing walkability and physical activity data. The participant data flow is shown in Figure 1.

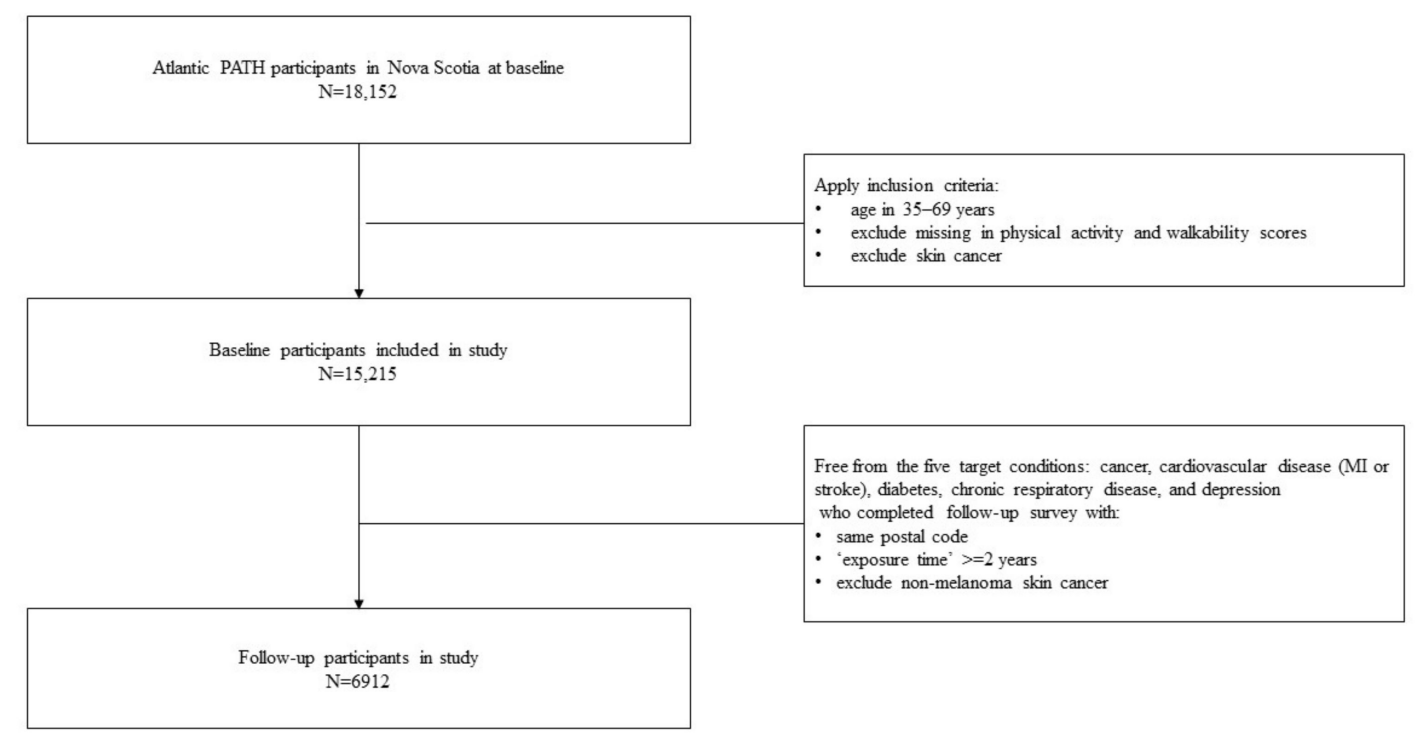

Figure 1. Participant data flow.

\subsection{Main Effects}

\subsubsection{Walkability}

Walkability was determined using the Canadian Active Living Environments (CAN-ALE) [16] dataset provided to Atlantic PATH by the Canadian Urban Environmental Health Research Consortium (CANUE). CANUE is a multidisciplinary collaboration of specialists focused on environmental exposures and population health [17]. In brief, CAN-ALE is a geography-based national metric of active living environments in Canada that can be used to facilitate direct comparisons between communities [16]. The ALE Index is a continuous measure of the favorability of the active living environment based on the summed z-scores of connectivity, dwelling density, and number of points of interest/destinations (e.g., shops, parks, businesses). Using the CAN-ALE Index, categorical walkability quintiles ranging from 1 (low walkability) to 5 (high walkability) were generated for all participants. CAN-ALE measures were linked to Atlantic PATH participant data through a six-digit residential postal code.

\subsubsection{Physical Activity}

Physical activity was assessed using both the short and long form of the International Physical Activity Questionnaire (IPAQ) at baseline. In accordance with the IPAQ scoring protocol [18], data from both forms were used to calculate categorical (low, moderate, high) physical activity scores by sex-specific total metabolic expenditure (MET-minutes/week) tertiles [19]. Participants categorized as moderately active met any of the following three criteria: (1) engaged in $\geq 3$ days/week of vigorous-intensity activity of at least $20 \mathrm{~min} /$ day; or (2) $\geq 5$ days of moderate-intensity activity or walking at least $30 \mathrm{~min} /$ day; or (3) $\geq 5$ days of any combination of walking or moderate- or vigorous-intensity activities, achieving a minimum of 6 MET-minutes/week. High active participants met either of the following two criteria: (1) engaged in vigorous-intensity activity on at least 3 days/week, accumulating 1500 MET-minutes/week; or (2) 7 days of any combination of walking, or moderate- or vigorous-intensity activities, achieving a minimum of 3000 MET-minutes/week. Those categorized as low active were not sufficiently active to meet the levels of either moderate or high activity. 


\subsection{Outcomes}

\subsubsection{Baseline}

The prevalence of chronic disease at baseline was ascertained through self-report, and included asthma, cancer, chronic obstructive pulmonary disease (COPD), chronic bronchitis, emphysema, liver cirrhosis, chronic hepatitis, myocardial infarction, stroke, hypertension, diabetes type 1/2, irritable bowel disease or irritable bowel syndrome, psoriasis or eczema, multiple sclerosis, arthritis, lupus, osteoporosis, depression, and obesity (defined by BMI $\geq 30 \mathrm{~kg} / \mathrm{m}^{2}$ ). The five target chronic conditions (cancer-excluding skin cancer; CVD_including myocardial infarction and/or stroke; diabetes; chronic respiratory disease-including COPD, chronic bronchitis, and emphysema; and depression) were selected for analyses given their high prevalence in Canada and the Atlantic provinces. Multimorbidity was defined as $\geq 2$ self-reported chronic conditions.

\subsubsection{Follow-Up}

Target chronic conditions were defined as self-reported, new (i.e., not reported at baseline) conditions that were revealed at follow-up, and included cancer (excluding skin cancer), CVD (myocardial infarction or stroke), diabetes, chronic respiratory disease, depression, and multi-morbidity (as defined above).

\subsection{Covariables}

Baseline characteristics, including sex, age, daily servings of fruit and vegetables, alcohol consumption, and smoking status were ascertained from Atlantic PATH participants' baseline data.

\subsubsection{Daily Fruit and Vegetable Consumption}

Total daily servings of fruit and vegetables were assessed by the following two questions: (1) In a typical day, how many servings of vegetables do you eat? One serving is about $\frac{1}{2}$ cup or $125 \mathrm{~mL}$ of fresh, frozen, canned or cooked vegetables and (2) In a typical day, how many servings of fruit do you eat? One serving is about $\frac{1}{2}$ cup or $125 \mathrm{~mL}$ of fresh, frozen, or canned fruit. For the present study, the number of daily servings of fruits and vegetables was summed. The combined value was expressed as the overall number of daily servings of fruit and vegetables. Adequate fruit and vegetable intake were defined as consuming at least 5 servings of combined daily servings of fruits and vegetables [20].

\subsubsection{Alcohol Consumption}

Participants were asked to indicate whether they had ever consumed alcohol (yes/no). If yes, they were asked to indicate the average frequency of alcohol consumption over the last year. Based on participant response they were classified as abstainer (never consumed alcohol), occasional drinker ( $>0$ to $\leq 2-3$ times/month), regular drinker ( $\geq 1$ time/week to $\leq 2-3$ times/week), or habitual drinker ( $\geq 4-5$ times/week) [20].

\subsubsection{Smoking}

Participants were first asked if they had smoked at least 100 cigarettes in their lifetime. If yes, they were asked to indicate at what age they smoked their first whole cigarette, what their smoking behavior was at present, at what age they began daily smoking, how many cigarettes per day they smoke now (or did when a daily smoker), and for how many years they were a daily smoker. Participants were then categorized as non-smoker (never having smoked 100 cigarettes in their life or other tobacco products on a regular basis for at least six months), former smoker (reported having at least 100 cigarettes in their lifetime but no tobacco use in the previous 30 days), and current smoker (those who reported smoking at least 100 cigarettes in their lifetime and smoked within the past 30 days) [20]. 


\subsubsection{Material and Social Deprivation Score}

Material and social deprivation scores were obtained through partnership with CANUE [17]. The domains related to the material dimension reflect the proportion of people with no high school diploma, average household income, and employment rate. The domains related to the social dimension are the proportion of individuals who are separated, divorced, or widowed, the proportion of single-parent families, and the proportion of persons living alone [21,22]. Material and social deprivation quintiles were generated by ranking participants on material and social deprivation scores at baseline and follow-up. Data merging with Atlantic PATH was facilitated through six-digit residential postal code linkage.

\subsubsection{Rurality}

Rurality was ascertained from the CANUE data set. The geographic area variable was categorized as follows: (1) a large census metropolitan area (CMA; includes the three largest metropolitan areas in Canada-Montreal, Toronto, and Vancouver); (2) other CMA (defined as a core population greater than 100,000); (3) census agglomeration (CA; defined as having a core population between 10,000 and 100,000); and (4) rural (defined as all areas inside the CMA or CA that are not core or fringe).

\subsection{Analyses}

We assessed the baseline characteristics of study participants by walkability quintile, mean and standard deviation for continuous variables, and frequency and percentage for dichotomous/nominal variables (Table 1). We then applied adjusted (sex, age, material and social deprivation, geographical area, and self-reported physical activity, smoking behaviors, and alcohol and fruit and vegetable consumption) logistic regression models to investigate the associations of walkability and physical activity with each chronic disease and multimorbidity outcome. Walkability and physical activity were estimated simultaneously in the adjusted model. High walkability and high physical activity were set as reference levels (Table 2). We then repeated all baseline analyses on the follow-up population; note that only those participants who did not report any of the five target chronic conditions were included in the final analyses (Tables 3 and 4). Data management and analyses were performed with SAS statistical package version 9.4 (SAS Institute, Cary, North Carolina) and R package version 3.6.3 [23].

Table 1. Sociodemographic and health-related participant characteristics by walking quintile.

\begin{tabular}{|c|c|c|c|c|c|c|}
\hline \multirow[b]{2}{*}{ Variables } & \multicolumn{5}{|c|}{ Walkability Quintile (Based on ale_06 ${ }^{\text {a) }}$} & \multirow[b]{2}{*}{$p$-Value } \\
\hline & $\begin{array}{c}\text { Q1 (Least) } \\
n=3310\end{array}$ & $\begin{array}{c}\text { Q2 } \\
n=2798\end{array}$ & $\begin{array}{c}\text { Q3 } \\
n=3097\end{array}$ & $\begin{array}{c}\text { Q4 } \\
n=2983\end{array}$ & $\begin{array}{c}\text { Q5 (Most) } \\
n=3027\end{array}$ & \\
\hline Sex, N (\%) & & & & & & 0.7224 \\
\hline Male & $977(29.5)$ & $848(30.3)$ & $932(30.1)$ & $925(31)$ & $932(30.8)$ & \\
\hline Female & $2333(70.5)$ & $1950(69.7)$ & $2165(69.9)$ & $2058(69)$ & $2095(69.2)$ & \\
\hline Age (SD) & $53.0(9.2)$ & $53.7(9.0)$ & $53.0(9.0)$ & $53.2(9.2)$ & $53.8(9.0)$ & 0.0004 \\
\hline Age group, N (\%) & & & & & & 0.0008 \\
\hline $35-49$ & $1192(36)$ & $928(33.2)$ & $1100(35.5)$ & $1044(35)$ & $955(31.5)$ & \\
\hline $50-59$ & $1152(34.8)$ & $970(34.7)$ & $1126(36.4)$ & $1051(35.2)$ & $1120(37)$ & \\
\hline $60-69$ & $966(29.2)$ & $900(32.2)$ & $871(28.1)$ & $888(29.8)$ & $952(31.5)$ & \\
\hline Rurality ${ }^{b}, \mathrm{~N}(\%)$ & & & & & & $<0.0001$ \\
\hline No & $1453(43.9)$ & $2021(72.2)$ & $2423(78.2)$ & 2643 (88.6) & 2964 (97.9) & \\
\hline Yes & $1627(49.2)$ & 713 (25.5) & $565(18.2)$ & $272(9.1)$ & 45 (1.5) & \\
\hline Unknown & $230(6.9)$ & $64(2.3)$ & $109(3.5)$ & $68(2.3)$ & $18(0.6)$ & \\
\hline
\end{tabular}


Table 1. Cont.

\begin{tabular}{|c|c|c|c|c|c|c|}
\hline \multirow[b]{2}{*}{ Variables } & \multicolumn{5}{|c|}{ Walkability Quintile (Based on ale_06 ${ }^{\text {a }}$ ) } & \multirow[b]{2}{*}{$p$-Value ${ }^{\mathrm{f}}$} \\
\hline & $\begin{array}{c}\text { Q1 (Least) } \\
n=3310\end{array}$ & $\begin{array}{c}\text { Q2 } \\
n=2798\end{array}$ & $\begin{array}{c}\text { Q3 } \\
n=3097\end{array}$ & $\begin{array}{c}\mathrm{Q} 4 \\
n=2983\end{array}$ & $\begin{array}{c}\text { Q5 (Most) } \\
n=3027\end{array}$ & \\
\hline $\begin{array}{l}\text { Material deprivation }{ }^{c} \\
\text { (quintile), N (\%) }\end{array}$ & & & & & & $<0.0001$ \\
\hline Q1 (Least deprived) & $122(3.7)$ & $473(16.9)$ & $562(18.1)$ & $1056(35.4)$ & $1387(45.8)$ & \\
\hline Q2 & $397(12)$ & $503(18)$ & $675(21.8)$ & $535(17.9)$ & $632(20.9)$ & \\
\hline Q3 & $733(22.1)$ & $699(25)$ & $594(19.2)$ & $490(16.4)$ & $336(11.1)$ & \\
\hline Q4 & $1062(32.1)$ & $609(21.8)$ & 609 (19.7) & 375 (12.6) & $423(14)$ & \\
\hline Q5 (Most deprived) & $704(21.3)$ & $381(13.6)$ & $509(16.4)$ & $319(10.7)$ & $157(5.2)$ & \\
\hline Unknown & $292(8.8)$ & $133(4.8)$ & $148(4.8)$ & $208(7)$ & $92(3)$ & \\
\hline $\begin{array}{r}\text { Social deprivation }{ }^{\mathrm{d}} \\
\text { (quintile), } \mathrm{N}(\%)\end{array}$ & & & & & & $<0.0001$ \\
\hline Q1 (Least deprived) & $751(22.7)$ & $930(33.2)$ & $689(22.2)$ & $497(16.7)$ & $179(5.9)$ & \\
\hline Q2 & $1382(41.8)$ & $776(27.7)$ & $699(22.6)$ & $568(19)$ & $368(12.2)$ & \\
\hline Q3 & $538(16.3)$ & $521(18.6)$ & 479 (15.5) & $203(6.8)$ & $251(8.3)$ & \\
\hline$\widehat{\mathrm{Q}} 4$ & $308(9.3)$ & $422(15.1)$ & $742(24)$ & $887(29.7)$ & $900(29.7)$ & \\
\hline Q5 (Most deprived) & $39(1.2)$ & $16(0.6)$ & $340(11)$ & $620(20.8)$ & $1237(40.9)$ & \\
\hline Unknown & $292(8.8)$ & $133(4.8)$ & $148(4.8)$ & $208(7)$ & $92(3)$ & \\
\hline Physical activity ${ }^{\mathrm{e}}$ & & & & & & $<0.0001$ \\
\hline Low & $1080(32.6)$ & $863(30.8)$ & $1037(33.5)$ & $1082(36.3)$ & $1011(33.4)$ & \\
\hline Moderate & $1074(32.4)$ & $951(34)$ & 1019 (32.9) & $946(31.7)$ & $1082(35.7)$ & \\
\hline High & $1156(34.9)$ & $984(35.2)$ & $1041(33.6)$ & $955(32)$ & $934(30.9)$ & \\
\hline Smoking status & & & & & & 0.0072 \\
\hline Never smoked & $1599(48.3)$ & $1388(49.6)$ & $1514(48.9)$ & $1508(50.6)$ & $1573(52)$ & \\
\hline Former smoker & $1306(39.5)$ & $1146(41)$ & $1249(40.3)$ & $1175(39.4)$ & $1160(38.3)$ & \\
\hline Current smoker & $369(11.1)$ & $246(8.8)$ & $304(9.8)$ & $280(9.4)$ & $277(9.2)$ & \\
\hline Unknown & $36(1.1)$ & $18(0.6)$ & $30(1)$ & $20(0.7)$ & $17(0.6)$ & \\
\hline $\begin{array}{c}\text { Daily fruits/vegetable } \\
\text { intake }\end{array}$ & & & & & & 0.2942 \\
\hline$\leq 4$ & $1680(50.8)$ & 1445 (51.6) & $1600(51.7)$ & $1536(51.5)$ & $1639(54.1)$ & \\
\hline$\geq 5$ & $1551(46.9)$ & $1296(46.3)$ & $1421(45.9)$ & $1381(46.3)$ & $1325(43.8)$ & \\
\hline Unknown & $79(2.4)$ & $57(2)$ & $76(2.5)$ & $66(2.2)$ & $63(2.1)$ & \\
\hline Alcohol consumption & & & & & & $<0.0001$ \\
\hline Abstainer & $231(7)$ & $182(6.5)$ & $191(6.2)$ & $172(5.8)$ & $186(6.1)$ & \\
\hline Occasional drinker & $994(30)$ & $796(28.4)$ & $959(31)$ & $821(27.5)$ & $745(24.6)$ & \\
\hline Regular & $1389(42)$ & $1225(43.8)$ & $1379(44.5)$ & $1390(46.6)$ & $1306(43.1)$ & \\
\hline Habitual drinker & $522(15.8)$ & $486(17.4)$ & $446(14.4)$ & $491(16.5)$ & $676(22.3)$ & \\
\hline Unknown & $174(5.3)$ & $109(3.9)$ & $122(3.9)$ & $109(3.7)$ & $114(3.8)$ & \\
\hline
\end{tabular}

Note: Q1-Quintile 1 (low); Q2-Quintile 2; Q3-Quintile 3; Q4-Quintile 4; Q5-Quintile 5 (high); ${ }^{a}$ Active Living Environment (ALE) Index—sum of all z-scores (Atlantic Partnership for Tomorrow's Health (PATH) Cohort linked with Canadian Active Living Environments (Can-ALE) walkability data). The following variables were linked to Atlantic PATH data from the Canadian Urban Environmental Health Research Consortium. ${ }^{b}$ Based on MSD_06-Geographical area_Large census metropolitan area, other census metropolitan area, census agglomeration, and rural; ' Based on MSD_08-Deprivation index-Material factor score; ${ }^{d}$ Based on MSD_09-Deprivation index-Social factor score; ${ }^{\mathrm{e}}$ International Physical Activity Questionnaire (IPAQ) data from both long and short forms were used to calculate categorical (low, moderate, high) physical activity scores by sex-specific total metabolic expenditure (MET-minutes/week); ${ }^{\mathrm{f}}$ F-test for continuous variables and Chi-Square test for categorical variables. 
Table 2. Associations of walkability and physical activity with selected chronic conditions and multi-morbidity at baseline.

\begin{tabular}{|c|c|c|c|}
\hline \multirow{2}{*}{ Main Effects } & \multirow{2}{*}{ Cases } & \multicolumn{2}{|c|}{ Adjusted Model $^{\text {a }}$} \\
\hline & & OR $(95 \% \mathrm{CI})$ & $p$-Value \\
\hline \multicolumn{4}{|l|}{ Diabetes } \\
\hline \multicolumn{4}{|l|}{ Walkability quintile } \\
\hline Q1 (Least) & 208 & $1.11(0.83,1.47)$ & 0.493 \\
\hline Q2 & 146 & $0.98(0.74,1.3)$ & 0.8826 \\
\hline Q3 & 172 & $1.06(0.82,1.36)$ & 0.6681 \\
\hline Q4 & 168 & $1.15(0.9,1.46)$ & 0.2557 \\
\hline Q5 (Most) & 151 & 1 (Ref) & - \\
\hline \multicolumn{4}{|l|}{ Physical activity } \\
\hline Low & 365 & $1.78(1.5,2.12)$ & $<0.0001$ \\
\hline Moderate & 255 & $1.19(0.99,1.43)$ & 0.0669 \\
\hline High & 225 & 1 (Ref) & - \\
\hline \multicolumn{4}{|c|}{ Cancer (excluding skin cancer) } \\
\hline \multicolumn{4}{|c|}{ Walkability quintile } \\
\hline Q1 (Least) & 165 & $2.96(1.94,4.54)$ & $<0.0001$ \\
\hline Q2 & 53 & $1.27(0.81,2.01)$ & 0.3009 \\
\hline$\widehat{Q} 3$ & 84 & $1.8(1.2,2.69)$ & 0.0045 \\
\hline $\mathrm{Q} 4$ & 91 & $2.14(1.46,3.13)$ & $<0.0001$ \\
\hline Q5 (Most) & 43 & 1 (Ref) & - \\
\hline \multicolumn{4}{|l|}{ Physical activity } \\
\hline Low & 132 & $0.85(0.67,1.08)$ & 0.1738 \\
\hline Moderate & 143 & $0.93(0.73,1.17)$ & 0.5119 \\
\hline High & 161 & 1 (Ref) & - \\
\hline \multicolumn{4}{|l|}{ Depression } \\
\hline \multicolumn{4}{|l|}{ Walkability quintile } \\
\hline Q1 (Least) & 220 & $2.08(1.47,2.95)$ & $<0.0001$ \\
\hline Q2 & 56 & $0.77(0.52,1.15)$ & 0.1972 \\
\hline Q3 & 110 & $1.28(0.92,1.78)$ & 0.135 \\
\hline$\widehat{\mathrm{Q}} 4$ & 135 & $1.75(1.3,2.36)$ & 0.0002 \\
\hline Q5 (Most) & 76 & 1 (Ref) & - \\
\hline \multicolumn{4}{|l|}{ Physical activity } \\
\hline Low & 234 & $1.21(0.99,1.48)$ & 0.0579 \\
\hline Moderate & 173 & $0.93(0.75,1.15)$ & 0.4902 \\
\hline High & 190 & 1 (Ref) & - \\
\hline \multicolumn{4}{|l|}{ CVD (MI or Stroke) } \\
\hline \multicolumn{4}{|l|}{ Walkability quintile } \\
\hline Q1 (Least) & 98 & $1.27(0.85,1.90)$ & 0.2412 \\
\hline $\mathrm{Q} 2$ & 70 & $1.08(0.73,1.59)$ & 0.7127 \\
\hline Q3 & 77 & $1.05(0.74,1.50)$ & 0.7900 \\
\hline$\hat{\mathrm{Q}} 4$ & 74 & $1.04(0.74,1.45)$ & 0.8345 \\
\hline Q5 (Most) & 81 & 1 (Ref) & - \\
\hline \multicolumn{4}{|l|}{ Physical activity } \\
\hline Low & 149 & $1.26(0.98,1.61)$ & 0.0667 \\
\hline Moderate & 124 & $1.01(0.78,1.30)$ & 0.9487 \\
\hline High & 127 & 1 (Ref) & - \\
\hline \multicolumn{4}{|c|}{ Chronic respiratory conditions ${ }^{b}$} \\
\hline \multicolumn{4}{|l|}{ Walkability quintile } \\
\hline Q1 (Least) & 85 & $0.62(0.43,0.89)$ & 0.0102 \\
\hline Q2 & 106 & $1.01(0.73,1.4)$ & 0.9504 \\
\hline$\widehat{\mathrm{Q} 3}$ & 107 & $0.89(0.66,1.2)$ & 0.4476 \\
\hline $\mathrm{Q} 4$ & 103 & $0.91(0.68,1.2)$ & 0.4877 \\
\hline Q5 (Most) & 121 & 1 (Ref) & - \\
\hline
\end{tabular}


Table 2. Cont.

\begin{tabular}{|c|c|c|c|}
\hline \multirow{2}{*}{ Main Effects } & \multirow{2}{*}{ Cases } & \multicolumn{2}{|c|}{ Adjusted Model a } \\
\hline & & OR (95\% CI) & $p$-Value \\
\hline \multicolumn{4}{|l|}{ Physical activity } \\
\hline Low & 209 & $1.27(1.03,1.56)$ & 0.0244 \\
\hline Moderate & 140 & $0.82(0.66,1.03)$ & 0.0958 \\
\hline High & 173 & 1 (Ref) & - \\
\hline \multicolumn{4}{|l|}{ Multi-morbidity ${ }^{c}$} \\
\hline \multicolumn{4}{|l|}{ Walkability quintile } \\
\hline Q1 (Least) & 1261 & $1.01(0.88,1.17)$ & 0.8406 \\
\hline Q2 & 1008 & $0.99(0.87,1.13)$ & 0.8567 \\
\hline Q3 & 1175 & $1.08(0.96,1.22)$ & 0.2132 \\
\hline$\widehat{\mathrm{Q}} 4$ & 1137 & $1.14(1.02,1.28)$ & 0.0247 \\
\hline Q5 (Most) & 1074 & 1 (Ref) & - \\
\hline \multicolumn{4}{|l|}{ Physical activity } \\
\hline Low & 2084 & $1.35(1.24,1.47)$ & $<0.0001$ \\
\hline Moderate & 1762 & $1(0.92,1.08)$ & 0.9448 \\
\hline High & 1809 & 1 (Ref) & - \\
\hline
\end{tabular}

Notes: Q1-Quintile 1 (low); Q2-Quintile 2; Q3-Quintile 3; Q4-Quintile 4; Q5-Quintile 5 (high); the interaction term for physical activity and Active Living Environment (walkability) for each of the outcomes was tested in the model and were not significant at the 0.05 level; ${ }^{a}$ adjusted for sex, age, rurality, socioeconomic status (both material/social deprivation scores quintile), alcohol consumption, daily servings of fruit and vegetables, smoking status, and physical activity/walkability where appropriate; ${ }^{b}$ chronic obstructive pulmonary disease, emphysema, and chronic bronchitis; ${ }^{\mathrm{c}}$ number of all chronic conditions $\geq 2$.

Table 3. Sociodemographic and health-related participant characteristics categorized by walking quintile at follow-up.

\begin{tabular}{|c|c|c|c|c|c|c|}
\hline \multirow[b]{2}{*}{ Variables } & \multicolumn{5}{|c|}{ Walkability Quintile (Based on ale_06 ${ }^{\text {a }}$ ) } & \multirow[b]{2}{*}{$p$-Value ${ }^{\mathrm{f}}$} \\
\hline & $\begin{array}{l}\text { Q1 (Least) } \\
n=1505\end{array}$ & $\begin{array}{c}\mathrm{Q} 2 \\
n=1268\end{array}$ & $\begin{array}{c}\mathrm{Q} 3 \\
n=1375\end{array}$ & $\begin{array}{c}\mathrm{Q} 4 \\
n=1388\end{array}$ & $\begin{array}{l}\text { Q5 (Most) } \\
n=1374\end{array}$ & \\
\hline Sex, N (\%) & & & & & & 0.0352 \\
\hline Male & $399(26.5)$ & $371(29.3)$ & $413(30)$ & $442(31.8)$ & $405(29.4)$ & \\
\hline Female & $1106(73.5)$ & $897(70.7)$ & $962(70)$ & $946(68.2)$ & $971(70.6)$ & \\
\hline Age (SD) & $53.3(8.8)$ & $53.4(8.7)$ & $53.5(8.7)$ & $53.3(8.8)$ & $54.0(8.7)$ & \\
\hline Age group, N (\%) & & & & & & 0.4170 \\
\hline 35-49 & $512(34)$ & $419(33)$ & $455(33.1)$ & $480(34.6)$ & $417(30.3)$ & \\
\hline $50-59$ & $567(37.7)$ & $465(36.7)$ & $519(37.7)$ & $500(36)$ & $534(38.8)$ & \\
\hline $60-69$ & $426(28.3)$ & $384(30.3)$ & $401(29.2)$ & $408(29.4)$ & $425(30.9)$ & \\
\hline Rurality ${ }^{\mathrm{b}}, \mathrm{N}(\%)$ & & & & & & $<0.0001$ \\
\hline No & $649(43.1)$ & $910(71.8)$ & $1050(76.4)$ & $1219(87.8)$ & 1363 (99.1) & \\
\hline Yes & $730(48.5)$ & $326(25.7)$ & $262(19.1)$ & $143(10.3)$ & $6(0.4)$ & \\
\hline Unknown & $126(8.4)$ & $32(2.5)$ & $63(4.6)$ & $26(1.9)$ & $7(0.5)$ & \\
\hline $\begin{array}{l}\text { Material deprivation }{ }^{\mathrm{c}} \\
\text { (quintile), } \mathrm{N}(\%)\end{array}$ & & & & & & $<0.0001$ \\
\hline Q1 (Least deprived) & $64(4.3)$ & $232(18.3)$ & $263(19.1)$ & $507(36.5)$ & $669(48.6)$ & \\
\hline $\mathrm{Q} 2$ & $187(12.4)$ & $211(16.6)$ & $299(21.7)$ & $260(18.7)$ & $304(22.1)$ & \\
\hline Q3 & $337(22.4)$ & $331(26.1)$ & $259(18.8)$ & $275(19.8)$ & $120(8.7)$ & \\
\hline Q4 & $471(31.3)$ & $243(19.2)$ & $255(18.5)$ & $147(10.6)$ & $182(13.2)$ & \\
\hline Q5 (Most deprived) & $293(19.5)$ & $194(15.3)$ & $224(16.3)$ & $124(8.9)$ & $59(4.3)$ & \\
\hline Unknown & $153(10.2)$ & $57(4.5)$ & $75(5.5)$ & $75(5.4)$ & $42(3.1)$ & \\
\hline
\end{tabular}


Table 3. Cont.

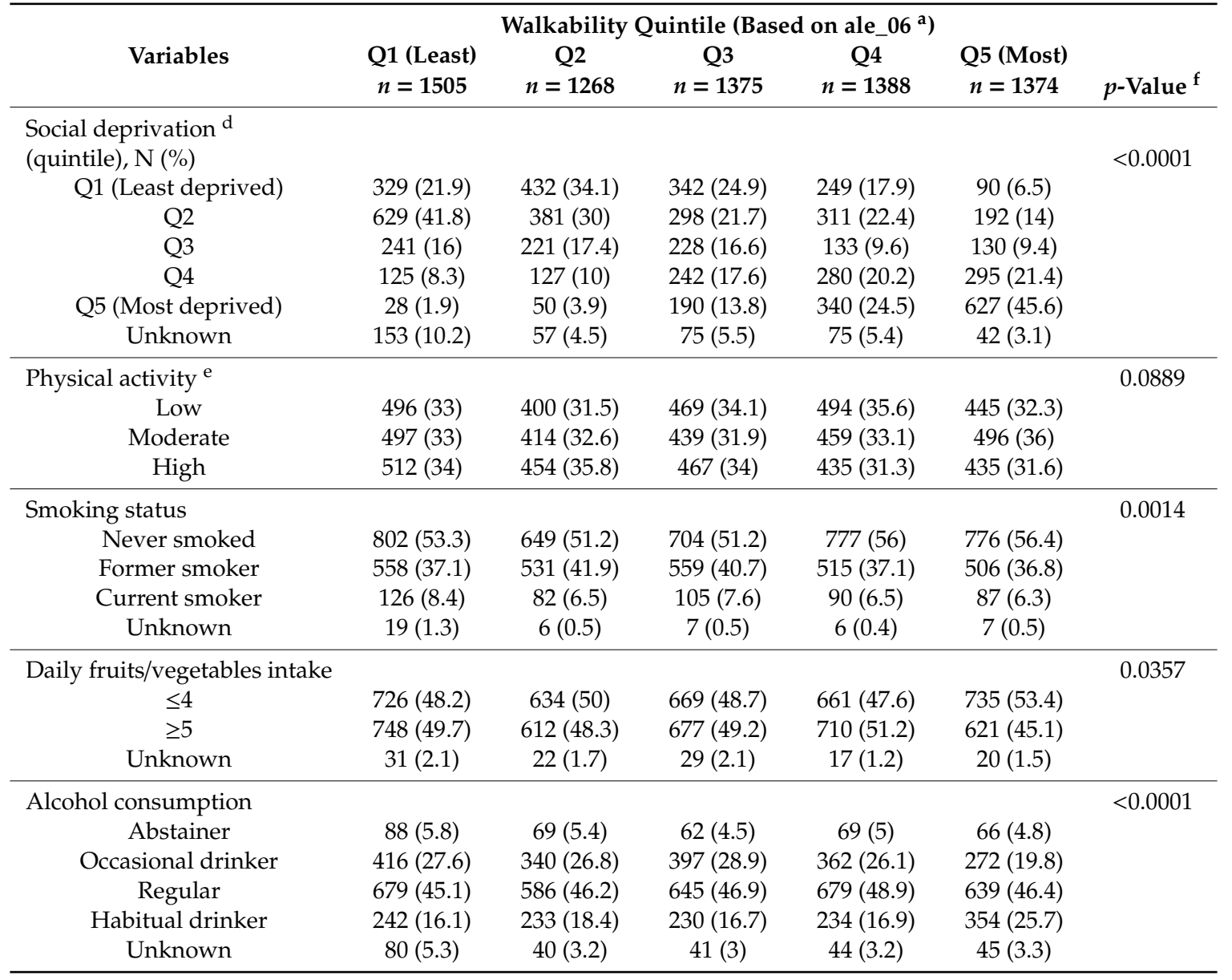

Note: Q1-Quintile 1 (low); Q2-Quintile 2; Q3-Quintile 3; Q4-Quintile 4; Q5-Quintile 5 (high); ${ }^{\text {a }}$ Active Living Environment (ALE) Index-sum of all z-scores (Atlantic Partnership for Tomorrow's Health (PATH) Cohort linked with Canadian Active Living Environments (Can-ALE) walkability data); The following variables were linked to the Atlantic PATH data from the Canadian Urban Environmental Health Research Consortium; ${ }^{b}$ Based on MSD_06-Geographical area-Large census metropolitan area, other census metropolitan area, census agglomeration, and rural; ' Based on MSD_08-Deprivation index_material factor score; d Based on MSD_09-Deprivation

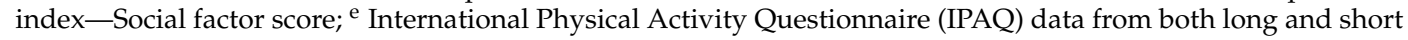
forms were used to calculate categorical (low, moderate, high) physical activity scores by sex-specific total metabolic expenditure (MET-minutes/week); ${ }^{\mathrm{f}}$ F-test for continuous variables and Chi-Square test for categorical variables.

Table 4. Associations of walkability and physical activity with selected chronic conditions and multi-morbidity at follow-up.

\begin{tabular}{lcccc}
\hline & & \multicolumn{2}{c}{ Adjusted Model $^{\text {a }}$} \\
\multicolumn{1}{c}{ Main Effects } & Cases & OR (95\% CI) & $p$-Value \\
\hline Diabetes & & & \\
Walkability quintile & & & \\
Q1 (Least) & 33 & $1.45(0.68,3.09)$ & 0.3323 \\
Q2 & 25 & $1.3(0.63,2.7)$ & 0.4743 \\
Q3 & 28 & $1.4(0.71,2.74)$ & 0.3331 \\
Q4 & 23 & $1.24(0.64,2.39)$ & 0.5288 \\
Q5 (Most) & 17 & $1($ Ref $)$ & - \\
Physical activity & & & \\
Low & 52 & $1.81(1.15,2.85)$ & 0.010 \\
Moderate & 43 & $1.45(0.91,2.31)$ & 0.1208 \\
High & 31 & $1($ Ref $)$ & - \\
\hline
\end{tabular}


Table 4. Cont.

\begin{tabular}{|c|c|c|c|}
\hline \multirow[b]{2}{*}{ Main Effects } & \multirow[b]{2}{*}{ Cases } & \multicolumn{2}{|c|}{ Adjusted Model a } \\
\hline & & OR $(95 \% \mathrm{CI})$ & $p$-Value \\
\hline \multicolumn{4}{|c|}{$\begin{array}{l}\text { Cancer } \\
\text { (non-melanoma skin cancer excluded) } \\
\text { Walkability quintile }\end{array}$} \\
\hline Q1 (Least) & 76 & $1.01(0.66,1.54)$ & 0.9669 \\
\hline Q2 & 64 & $0.88(0.59,1.31)$ & 0.5304 \\
\hline Q3 & 77 & $0.96(0.67,1.38)$ & 0.817 \\
\hline $\mathrm{Q} 4$ & 80 & $1.01(0.72,1.41)$ & 0.9654 \\
\hline Q5 (Most) & 81 & 1 (Ref) & - \\
\hline \multicolumn{4}{|l|}{ Physical activity } \\
\hline Low & 142 & $1.41(1.09,1.83)$ & 0.0092 \\
\hline Moderate & 126 & $1.18(0.9,1.54)$ & 0.2232 \\
\hline High & 110 & 1 (Ref) & - \\
\hline \multicolumn{4}{|l|}{ Depression } \\
\hline \multicolumn{4}{|l|}{ Walkability quintile } \\
\hline Q1 (Least) & 6 & $1.11(0.31,4.02)$ & 0.8683 \\
\hline Q2 & 4 & $0.58(0.15,2.22)$ & 0.4241 \\
\hline Q3 & 8 & $1(0.33,3.02)$ & 0.9975 \\
\hline Q4 & 7 & $0.81(0.28,2.33)$ & 0.6988 \\
\hline Q5 (Most) & 9 & 1 (Ref) & - \\
\hline \multicolumn{4}{|l|}{ Physical activity } \\
\hline Low & 13 & $1.29(0.55,3.06)$ & 0.5574 \\
\hline Moderate & 12 & $1.24(0.52,2.96)$ & 0.6336 \\
\hline High & 9 & 1 (Ref) & - \\
\hline \multicolumn{4}{|l|}{ CVD (MI or Stroke) } \\
\hline \multicolumn{4}{|l|}{ Walkability quintile } \\
\hline Q1 (Least) & 5 & $0.52(0.14,1.97)$ & 0.3355 \\
\hline Q2 & 12 & $1.63(0.57,4.65)$ & 0.3635 \\
\hline Q3 & 14 & $1.61(0.63,4.16)$ & 0.3232 \\
\hline $\mathrm{Q} 4$ & 8 & $0.92(0.34,2.48)$ & 0.866 \\
\hline Q5 (Most) & 9 & 1 (Ref) & - \\
\hline \multicolumn{4}{|l|}{ Physical activity } \\
\hline Low & 16 & $0.74(0.39,1.42)$ & 0.3656 \\
\hline Moderate & 9 & $0.4(0.18,0.87)$ & 0.0213 \\
\hline High & 23 & 1 (Ref) & - \\
\hline \multicolumn{4}{|c|}{ Chronic respiratory disease ${ }^{b}$} \\
\hline \multicolumn{4}{|l|}{ Walkability quintile } \\
\hline Q1 (Least) & 11 & $1.7(0.51,5.7)$ & 0.3863 \\
\hline Q2 & 9 & $1.8(0.58,5.64)$ & 0.3101 \\
\hline Q3 & 11 & $1.59(0.56,4.51)$ & 0.3866 \\
\hline$\widehat{\mathrm{Q}} 4$ & 10 & $1.63(0.6,4.44)$ & 0.3429 \\
\hline Q5 (Most) & 7 & 1 (Ref) & - \\
\hline \multicolumn{4}{|l|}{ Physical activity } \\
\hline Low & 22 & $1.51(0.79,2.89)$ & 0.2175 \\
\hline Moderate & 10 & $0.63(0.29,1.4)$ & 0.2613 \\
\hline High & 16 & 1 (Ref) & - \\
\hline \multicolumn{4}{|l|}{ Multi-morbidity ${ }^{c}$} \\
\hline \multicolumn{4}{|l|}{ Walkability quintile } \\
\hline Q1 (Least) & 554 & $0.95(0.78,1.17)$ & 0.6413 \\
\hline Q2 & 493 & $1.08(0.89,1.31)$ & 0.417 \\
\hline Q3 & 551 & $1.12(0.94,1.34)$ & 0.2035 \\
\hline $\mathrm{Q} 4$ & 548 & $1.17(0.99,1.38)$ & 0.0642 \\
\hline Q5 (Most) & 492 & 1 (Ref) & - \\
\hline
\end{tabular}


Table 4. Cont

\begin{tabular}{cccc}
\hline & \multicolumn{3}{c}{ Adjusted Model $^{\mathbf{a}}$} \\
Main Effects & Cases & OR $\mathbf{( 9 5 \% \mathbf { C I } )}$ & $\boldsymbol{p}$-Value \\
\hline Physical activity & & & \\
Low & 958 & $1.26(1.11,1.42)$ & 0.0002 \\
Moderate & 817 & $0.94(0.83,1.06)$ & 0.3143 \\
High & 863 & 1 (Ref) & - \\
\hline
\end{tabular}

Notes: Q1-Quintile 1 (low); Q2-Quintile 2; Q3-Quintile 3; Q4—Quintile 4; Q5-Quintile 5 (high); the interaction term for physical activity and the Active Living Environment (walkability) for each of the outcomes was tested in the model, and were not significant at the 0.05 level; ${ }^{a}$ adjusted for sex, age, rurality, socioeconomic status (both material/social deprivation scores quintile), alcohol consumption, daily servings of fruit and vegetables, smoking status, and physical activity/walkability where appropriate; ${ }^{b}$ chronic obstructive pulmonary disease, emphysema, and chronic bronchitis; ${ }^{\mathrm{c}}$ number of all chronic conditions $\geq 2$.

\section{Results}

\subsection{Cross-Sectional Study Findings}

Participant characteristics are presented by walking quintiles in Table 1. Participants with a high level of walkability were more likely to be more socially deprived, but less likely to be materially deprived. Rural participants were less likely to live in a highly walkable neighborhood and high physical activity levels were found to decrease with higher neighborhood walkability.

Table 2 displays the results for the associations between walkability and physical activity with selected health conditions and multimorbidity. Low levels of physical activity were significantly associated with higher odds of diabetes (OR 1.78, 95\% CI 1.5-2.12, $p<0.0001$ ). No significant association was found for low walkability (OR 1.11,95\% CI 0.83-1.47, $p=0.493$ ).

With the exception of the second quintile, low to moderate levels of walkability were significantly associated with higher odds of cancer. Of note, higher walkability levels (quintile 4) were also shown to relate to significantly higher odds of cancer (OR 2.14, 95\% CI 1.46-3.13, $p<0.0001$, respectively). Physical activity was not significantly associated with higher odds of cancer.

Higher odds of depression were found for low levels of both walkability (OR 2.08, 95\% CI 1.47-2.95, $p<0.0001$ ) and physical activity (OR 1.21, 95\% CI 0.99-1.48, $p=0.0579$ ). Similar to the association with cancer, higher walkability (quintile 4 ) was shown to relate to significantly higher odds of depression (OR 1.75, 95\% CI 1.3-2.36, $p=0.0002$ ).

No significant associations were found for either low walkability or physical activity and CVD.

Compared to those in highly walkable neighborhoods, residents in the least walkable neighborhoods were significantly less likely to have chronic respiratory disease $(\mathrm{OR}=0.62,95 \% \mathrm{CI}$ $0.43-0.89, p=0.0102)$. Participants with low levels of physical activity were significantly more likely to have chronic respiratory disease (OR $=1.27,95 \%$ CI 1.03-1.56, $p=0.0244)$.

Low physical activity, but not walkability, was significantly associated with multimorbidity (OR 1.35, 95\% CI 1.24-1.47, $p<0.0001$ ). As noted with both cancer and depression, higher walkability (quintile 4) was shown to relate to significantly higher odds of multimorbidity (OR 1.14, 95\% CI $1.02-1.28, p=0.0247$ respectively).

\subsection{Prospective Study Findings}

Table 3 shows the sociodemographic and health characteristics of the 6912 Nova Scotia participants who completed the follow-up questionnaire, who were free from the five target chronic conditions (i.e., cancer, CVD, diabetes, depression, and chronic respiratory disease) and were residing within the same postal code for at least for 2 years. The findings mirror those of the baseline cross-sectional findings where participants with the highest level of walkability were most likely to be socially deprived, but least likely to be materially deprived. Rural residents were again shown to have the least walkable neighborhoods. No clear trend was noted for physical activity and walkability at follow-up. 
Table 4 displays the results for the associations between walkability and baseline physical activity with selected health conditions and multimorbidity at follow-up. Low levels of baseline physical activity were significantly associated with higher odds of diabetes (OR 1.81, 95\% CI 1.15-2.85, $p=0.010$ ). No significant associations were found for walkability.

Low levels of baseline physical activity were associated with higher odds of cancer (OR 1.41, 95\% CI 1.09-1.83, $p=0.0092$ ). No significant associations were found with lower levels of walkability and odds of cancer.

No significant associations between either low walkability or levels of baseline physical activity and odds of depression were found.

No significant association was found between walkability and CVD. Moderate levels of baseline physical activity were shown to be significantly associated with decreased odds of CVD (OR 0.40, 95\% CI 0.18-0.87, $p=0.0213$ ).

There were no significant findings for the relationship between walkability or physical activity and chronic respiratory disease.

Low levels of baseline physical activity, but not low walkability, were significantly associated with higher odds of multimorbidity (OR 1.26, 95\% CI 1.11-1.42, $p=0.0002$ ).

\section{Discussion}

Several modifiable risk behaviors have been associated with the physical environment. For example, a growing body of research has shown that the physical environment plays an important role in supporting an active lifestyle through the collective availability of activity-friendly neighborhood characteristics (i.e., walkability) across several cultural contexts [11,24-26]. As physical activity has been well-established to reduce chronic disease risk [27], residents of highly walkable communities should, by extension, present with better health outcomes [28].

While previous studies have observed a positive association between neighborhood walkability and physical activity $[11,25,29,30]$, these findings are inconsistent with our own. We did not find a positive linear trend in either low or moderate levels of physical activity and walkability at baseline. A notable finding was the downward linear trend in high levels of physical activity with higher walkability at baseline. No clear association was found between any level of physical activity and walkability at follow-up. Although it is not clear why this relationship/lack of relationship emerged, it is well-established that physical activity behaviors are influenced by a complex interplay of intra- (e.g., social support) and inter-personal (e.g., knowledge, motivation), cultural, economic, and environmental factors. For example, residential self-selection has been shown to influence the impact of the built environment on an individual's or group's physical activity behaviors [30,31]. Those more inclined to walk for transportation (i.e., intra-personal influence) may seek out and reside in neighborhoods which provide greater access to amenities within walking distance. Those who are less inclined to walk for any purpose are not likely to choose a neighborhood based on perceived walkability [30]. Research has also shown that some neighborhood characteristics are more important than others with respect to supporting different types of walking (i.e., walking for transportation vs. walking for recreation) [32]. These findings are consistent with the work of others who have shown that walkability is positively correlated with walking for transport but shows no or negative associations with recreational physical activity [28]. Thus, the relationship between neighborhood walkability and physical activity can be influenced by the characteristics of the environment itself, an individual's preference for physical activity, or both [31].

The findings from the cross-sectional data are partially consistent with others and suggest that compared to individuals in the highest walkability category, participants with the lowest neighborhood walkability were more likely to have reported a pre-existing history of cancer and depression [8]. Those residing in low walkable neighborhoods were less likely to report a pre-existing history of a chronic respiratory disease. While we are only able to speculate, the decreased prevalence of chronic respiratory conditions may be partially attributable to the reduced adverse impacts of exposure to air 
pollution, which are typically found in more walkable urban centers [33,34]. Notably, participants residing in the higher walkability categories (quintiles 3 and/or 4) were more likely to report having cancer, depression and multimorbidity. These incongruent findings suggest that while activity-friendly neighborhoods may support health-promoting behaviors (e.g., physical activity), the benefits are limited to those who are willing to engage in the behavior(s).

Compared to those with the highest level of physical activity, participants with low physical activity were more likely to have a pre-existing history of diabetes, chronic respiratory disease, and multimorbidity. These findings highlight the importance of physical activity in both the primary and secondary prevention of chronic disease [27].

At follow-up, no significant associations were found between neighborhood walkability and incident chronic disease or multimorbidity. As walkability was not linearly associated with physical activity in our models, our findings reinforce the notion that if you build it, they may or may not come. Consistent with the work of others, the protective health benefits of neighborhood walkability appear to be at least partially mediated by physical activity levels [10].

Overall, the findings from the prospective study suggest a stronger relationship between physical activity and chronic disease. Specifically, participants with low activity were significantly more likely to be diagnosed with diabetes and cancer. Less active participants were also significantly more likely to have multiple chronic conditions. These findings are again in alignment with the growing body of evidence which demonstrates a decreased risk of chronic illness with increasing levels of physical activity [27].

\section{Strengths and Limitations}

To the best of our knowledge, this is the first Canadian study to examine the association between walkability and cancer, depression and multimorbidity, and is the first study to consider the association between standardized measures of walkability and health outcomes in Atlantic Canada. This study utilized a large sample of Nova Scotia participants and accounted for sociodemographic moderators (e.g., age, sex, material and social deprivation, rurality), as well as controlling for confounding factors (e.g., smoking history, alcohol consumption, physical activity). A significant strength of the study was our ability to include both prevalent and incident cases of five common chronic diseases and multimorbidities by utilizing both baseline and follow-up data.

We do acknowledge the limitations of our study, such as being limited by self-reported physical activity and chronic disease incidence/prevalence data (i.e., data subject to social biases and/or personal knowledge and willingness to report). We were also not able to assess disease severity, which may impact activity levels. Further, the cross-sectional design is limited by possible reverse causality.

Finally, there are limitations with the self-reported IPAQ data, though it is a validated and widely used measure of physical activity [35]. As we combined data from both the long- and short-form of the IPAQ measure, we used an aggregate measure of physical activity, which was categorized as low, moderate, and high. In doing so, we were not able to examine domain-specific associations between neighborhood walkability and physical activity levels. Further, as data on physical activity were not collected at the first follow-up, we were constrained to a single, self-reported measure of baseline physical activity across all analyses. Future research will include new reports of physical activity and health outcomes collected as part of the prospective nature of Atlantic PATH. We will also consider regional differences in walkability, physical activity and chronic disease by expanding this work to the other regional CanPath cohorts across Canada.

\section{Conclusions}

In sum, we did not find a positive association between walkability and chronic disease incidence in the current study. However, our data provide evidence for the health protective benefits of higher levels of physical activity, and a reduction in prevalence of some chronic diseases in more walkable communities. Thus, highly walkable neighborhoods may confer health benefits for those residents 
willing and able to engage in physical activity. An additional study is needed in order to better understand the interactional effects of both individual-level influences and the broader social and cultural climate on population-level behavior change. Moreover, there is a need for a culture shift whereby unhealthy behaviors, such as physical inactivity, are denormalized [36]. Environments which are activity-friendly and improve access to recreational resources, such as parks, playgrounds, and green space, can reduce perceived barriers to activity and can enforce activity as a social and cultural norm [37].

Author Contributions: Conceptualization, M.R.K., V.D., S.A.G. and E.S.; formal analysis, Y.C.; funding acquisition, T.J.B.D.; methodology, M.R.K., Y.C., S.A.G., E.S. and T.J.B.D.; supervision, T.J.B.D.; writing-Original draft, M.R.K.; writing-Review and editing, M.R.K., Y.C., V.D., S.A.G., E.S. and T.J.B.D. All authors have read and agreed to the published version of the manuscript.

Funding: The data used in this research were made available by the Atlantic Partnership for Tomorrow's Health (Atlantic PATH) study, which is the Atlantic Canada regional component of the Canadian Partnership for Tomorrow's Health (CanPath, formerly known as the Canadian Partnership for Tomorrow Project). Production of this study has been made possible through financial support from the Canadian Partnership Against Cancer and by Health Canada. The views expressed herein represent the views of the authors and do not necessarily represent the views of Health Canada.

Acknowledgments: We would like to thank the Atlantic PATH participants who donated their time and personal health history to this project. We would also like to thank the Atlantic PATH team members for data collection and management. Finally, we would like to acknowledge that the environmental exposure data was obtained through linkage with Canadian Urban Environment Health Research Consortium (CANUE).

Conflicts of Interest: The authors declare no conflict of interest. The study sponsors had no role in the design, execution, interpretation, or writing of the study.

\section{References}

1. Center for Surveillance and Applied Research, Public Health Agency of Canada. Canadian Chronic Disease Indicators Data Tool, 2019 ed.; Public Health Agency of Canada: Ottawa, ON, Canada, 2019. Available online: https://health-infobase.canada.ca/ccdi/data-tool/?Dom=1 (accessed on 1 September 2019).

2. Manuel, D.G.; Perez, R.; Sanmartin, C.; Taljaard, M.; Hennessy, D.; Wilson, K.; Tanuseputro, P.; Manson, H.; Bennett, C.; Tuna, M.; et al. Measuring Burden of Unhealthy Behaviours Using a Multivariable Predictive Approach: Life Expectancy Lost in Canada Attributable to Smoking, Alcohol, Physical Inactivity, and Diet. PLoS Med. 2016, 13, e1002082. [CrossRef]

3. Branchard, B.; Deb-Rinker, P.; Dubois, A.; Lapointe, P.; O'Donnell, S.; Pelletier, L.; Williams, G. At-a-glance-How Healthy are Canadians? A brief update. Health Promot. Chronic Dis. Prev. Can. 2018, 38, 385-387. [CrossRef]

4. Nova Scotia Health Profile. 2015. Available online: https:/novascotia.ca/dhw/publichealth/documents/ Population-Health-Profile-Nova-Scotia.pdf (accessed on 1 September 2020).

5. Bauer, U.E.; Briss, P.A.; Goodman, R.A.; Bowman, B.A. Prevention of chronic disease in the 21st century: Elimination of the leading preventable causes of premature death and disability in the USA. Lancet 2014, 384, 45-52. [CrossRef]

6. Schmidt, H. Chronic disease prevention and health promotion. In Public Health Ethics: Cases Spanning the Globe; Springer: Berlin/Heidelberg, Germany, 2016; pp. 137-176.

7. Hills, A.P.; Farpour-Lambert, N.J.; Byrne, N.M. Precision medicine and healthy living: The importance of the built environment. Prog. Cardiovasc. Dis. 2019, 62, 34-38. [CrossRef] [PubMed]

8. McCormack, G.R.; Cabaj, J.; Orpana, H.; Lukic, R.; Blackstaffe, A.; Goopy, S.; Hagel, B.; Keough, N.; Martinson, R.; Chapman, J.; et al. Evidence synthesis A scoping review on the relations between urban form and health: A focus on Canadian quantitative evidence. Health Promot. Chronic Dis. Prev. Can. 2019, 39, 187-200. [CrossRef] [PubMed]

9. Méline, J.; Chaix, B.; Pannier, B.; Ogedegbe, G.; Trasande, L.; Athens, J.K.; Duncan, D.T. Neighborhood walk score and selected Cardiometabolic factors in the French RECORD cohort study. BMC Public Health 2017, 17, 960. [CrossRef] 
10. Chandrabose, M.; Cerin, E.; Mavoa, S.; Dunstan, D.W.; Carver, A.; Turrell, G.; Owen, N.; Giles-Corti, B.; Sugiyama, T. Neighborhood walkability and 12-year changes in cardio-metabolic risk: The mediating role of physical activity. Int. J. Behav. Nutr. Phys. Act. 2019, 16, 86. [CrossRef]

11. Colley, R.C.; Christidis, T.; Michaud, I.; Tjepkema, M.; Ross, N.A. The association between walkable neighbourhoods and physical activity across the lifespan. Health Rep. 2019, 30, 3-13.

12. Dummer, T.J.; Awadalla, P.; Boileau, C.; Craig, C.; Fortier, I.; Goel, V.; Hicks, J.M.; Jacquemont, S.; Knoppers, B.M.; Le, N.; et al. The Canadian Partnership for Tomorrow Project: A pan-Canadian platform for research on chronic disease prevention. Can. Med. Assoc. J. 2018, 190, E710-E717. [CrossRef]

13. Sweeney, E.; Cui, Y.; Declercq, V.; Devichand, P.; Forbes, C.; Grandy, S.; Hicks, J.M.T.; Keats, M.; Parker, L.; Thompson, D.; et al. Cohort Profile: The Atlantic Partnership for Tomorrow's Health (Atlantic PATH) Study. Int. J. Epidemiol. 2017, 46, 1762i-1763i. [CrossRef]

14. Statistics Canada. Table 17-10-0009-01 Population Estimates, Quarterly. Available online: https://www150. statcan.gc.ca/t1/tbl1/en/tv.action?pid=1710000901 (accessed on 1 September 2020).

15. Statistics Canada. Table 17-10-0139-01 Population Estimates, July 1, by Census Division, 2016 Boundaries. Available online: https://www150.statcan.gc.ca/t1/tbl1/en/tv.action?pid=1710013901 (accessed on 1 September 2020).

16. Hermann, T.; Gleckner, W.; Wasfi, R.A.; Thierry, B.; Kestens, Y.; Ross, N.A. A pan-Canadian measure of active living environments using open data. Public Health Rep. 2019, 30, 16-25.

17. Brook, J.R.; Setton, E.M.; Seed, E.; Shooshtari, M.; Doiron, D.; CANUE-The Canadian Urban Environmental Health Research Consortium. The Canadian Urban Environmental Health Research Consortium-A protocol for building a national environmental exposure data platform for integrated analyses of urban form and health. BMC Public Health 2018, 18, 114. [CrossRef] [PubMed]

18. IPAQ Scoring Protocol. Available online: https://sites.google.com/site/theipaq/scoring-protocol (accessed on 19 November 2020).

19. Forbes, C.C.; Yu, Z.M.; Cui, Y.; Declercq, V.; Grandy, S.A.; Parker, L.; Sweeney, E.; Dummer, T.J.B.; Keats, M.R. Rural-Urban Disparities in Total Physical Activity, Body Composition, and Related Health Indicators: An Atlantic PATH Study. J. Rural Health 2020, 36, 111-119. [CrossRef] [PubMed]

20. Keats, M.R.; Cui, Y.; Declercq, V.; Grandy, S.A.; Sweeney, E.; Dummer, T.J.B. Burden of multimorbidity and polypharmacy among cancer survivors: A population-based nested case-control study. Support. Care Cancer 2020, 1-11. [CrossRef] [PubMed]

21. Saint-Jacques, N.; Lee, J.S.W.; Brown, P.; Stafford, J.; Parker, L.; Dummer, T.J.B. Small-area spatio-temporal analyses of bladder and kidney cancer risk in Nova Scotia, Canada. BMC Public Health 2016, 16, 1-17. [CrossRef]

22. Pampalon, R.; Hamel, D.; Gamache, P.; Philibert, M.D.; Raymond, G.; Simpson, A. An area-based material and social deprivation index for public health in Québec and Canada. Can. J. Public Health 2012, 103 (Suppl. 2), S17-S22. [CrossRef]

23. The R Project for Statistical Computing. Available online: https://www.r-project.org/ (accessed on 19 November 2020).

24. Chen, B.-I.; Hsueh, M.-C.; Rutherford, R.; Park, J.-H.; Liao, Y. The associations between neighborhood walkability attributes and objectively measured physical activity in older adults. PLoS ONE 2019, 14, e0222268. [CrossRef]

25. Hajna, S.; Ross, N.A.; Brazeau, A.-S.; Belisle, P.; Joseph, L.; Dasgupta, K. Associations between neighbourhood walkability and daily steps in adults: A systematic review and meta-analysis. BMC Public Health 2015, 15, 768. [CrossRef]

26. Sundquist, K.; Eriksson, U.; Kawakami, N.; Skog, L.; Ohlsson, H.; Arvidsson, D. Neighborhood walkability, physical activity, and walking behavior: The Swedish Neighborhood and Physical Activity (SNAP) study. Soc. Sci. Med. 2011, 72, 1266-1273. [CrossRef]

27. Warburton, D.E.R.; Bredin, S.S.D. Health benefits of physical activity: A systematic review of current systematic reviews. Curr. Opin. Cardiol. 2017, 32, 541-556. [CrossRef]

28. Farkas, B.; Wagner, D.J.; Nettel-Aguirre, A.; Friedenreich, C.; McCormack, G.R. Evidence synthesis-A systematized literature review on the associations between neighbourhood built characteristics and walking among Canadian adults. Health Promot. Chronic Dis. Prev. Can. 2019, 39, 1-14. [CrossRef] [PubMed] 
29. McCormack, G.R. Neighbourhood built environment characteristics associated with different types of physical activity in Canadian adults. Health Promot. Chronic Dis. Prev. Can. 2017, 37, 175-185. [CrossRef] [PubMed]

30. McCormack, G.R.; Shiell, A. In search of causality: A systematic review of the relationship between the built environment and physical activity among adults. Int. J. Behav. Nutr. Phys. Act. 2011, 8, 125. [CrossRef] [PubMed]

31. Boone-Heinonen, J.; Gordon-Larsen, P.; Guilkey, D.K.; Jacobs, D.R.; Popkin, B.M. Environment and physical activity dynamics: The role of residential self-selection. Psychol. Sport Exerc. 2011, 12, 54-60. [CrossRef] [PubMed]

32. Owen, N.; Cerin, E.; Leslie, E.; Du Toit, L.; Coffee, N.; Frank, L.D.; Bauman, A.E.; Hugo, G.; Saelens, B.E.; Sallis, J.F. Neighborhood Walkability and the Walking Behavior of Australian Adults. Am. J. Prev. Med. 2007, 33, 387-395. [CrossRef]

33. Sarkar, C.; Zhang, B.; Ni, M.; Kumari, S.; Bauermeister, S.; Gallacher, J.; Webster, C. Environmental correlates of chronic obstructive pulmonary disease in 96779 participants from the UK Biobank: A cross-sectional, observational study. Lancet Planet. Health 2019, 3, e478-e490. [CrossRef]

34. Doiron, D.; Setton, E.M.; Shairsingh, K.; Brauer, M.; Hystad, P.; Ross, N.A.; Brook, J.R. Healthy built environment: Spatial patterns and relationships of multiple exposures and deprivation in Toronto, Montreal and Vancouver. Environ. Int. 2020, 143, 106003. [CrossRef]

35. Keats, M.R.; Cui, Y.; Declercq, V.; Dummer, T.J.; Forbes, C.C.; Grandy, S.A.; Hicks, J.; Sweeney, E.; Yu, Z.; Parker, L. Multimorbidity in Atlantic Canada and association with low levels of physical activity. Prev. Med. 2017, 105, 326-331. [CrossRef]

36. Yun, L.; Vanderloo, L.; Berry, T.R.; Latimer-Cheung, A.E.; O'Reilly, N.; Rhodes, R.E.; Spence, J.C.; Tremblay, M.S.; Faulkner, G. Assessing the social climate of physical (in)activity in Canada. BMC Public Health 2018, 18, 1301. [CrossRef]

37. Public Health Agency of Canada. A Common Vision for Increasing Physical Activity and Reducing Sedentary Living in Canada: Let's Get Moving. Available online: https://www.canada.ca/en/public-health/services/ publications/healthy-living/lets-get-moving.html (accessed on 19 November 2020).

Publisher's Note: MDPI stays neutral with regard to jurisdictional claims in published maps and institutional affiliations.

(C) 2020 by the authors. Licensee MDPI, Basel, Switzerland. This article is an open access article distributed under the terms and conditions of the Creative Commons Attribution (CC BY) license (http://creativecommons.org/licenses/by/4.0/). 